\title{
Centralized and Decentralized of Quadruple Tank Process
}

\author{
P.Srinivasarao \\ Research scholar \\ Dr.M.G.R.University \\ Chennai, India
}

\author{
P.Subbaiah, PhD. \\ Prof of Dhanalaxmi college of Engineering \\ Thambaram \\ Chennai, India
}

\begin{abstract}
In this paper, Model Predictive Control of Quadruple tank process for centralized and decentralized method is proposed. Multi Input Multi Output (MIMO) processes are inherently more complex than Single Input Single Output (SISO) process because process interactions occur between controlled and manipulated variables. This problem can be solved using centralized and decentralized controllers. Model Predictive Control (MPC) technique can be applied to both centralized and decentralized of Quadruple tank processes. It consists of four inter connected water tanks and two pumps as shown in fig.1. A general MPC control is presented and different approaches taken for the different aspects of the calculations are described. It is shown that MPC control is more stable, responsive and robust.
\end{abstract}

\section{Keywords}

MIMO, MPC, Quadruple Tank Process, Robust.

\section{INTRODUCTION}

The major purpose is to solve the control problem for static as well as dynamic systems using model predictive control (MPC). Here, MPC is a more advanced control technique for predetermined output along with tuning the parameters such as prediction and control horizons and control weights. It can handle multivariable processes, difficult multivariable control problems that include inequality constraint [11]. Decentralized [1], [2] and centralized [3] model predictive control addresses the problem of controlling a multivariable dynamic process. Decentralized process, composed by iterating subsystems and subject to constraints, in computation and efficient way of communication, compared to a centralized MPC setup. In DMPC, it eliminates loop interaction between controlled and manipulated variables. The process is called the quadruple-tank process and consists of four interconnected water tanks and two pumps. The system is shown in Figure 1. The inputs are the voltages to the two pumps and the outputs are the water levels in the lower two tanks. The quadruple-tank process can easily be built by using two double-tank processes. The linearized model of the quadruple-tank process has a multivariable zero, which can be located on either the left or the right half-plane by simply changing a valve. Quadruple tank contains transmission zeros, which can vary from left half plane (minimum phase) to right half plane (non-minimum phase) depending on the ratio of the flow to upper and lower tanks.

The step response of the quadruple tank system using MPC controllers with different tuning parameters is obtained and compared to the step response of decoupled control strategy [1] ,comparative study [2] and linear and nonlinear model predictive control of Quadruple tank process [3].
This paper is organized as follows: section 2 gives description of four tank process. The controller design for four tanks and proposed MPC for stability is explained in $3 \& 4$. The analysis and simulation results are given in section 5. Finally the conclusion is given in 6 .

\section{DESCRITON OF FOUR TANK PROCESS}

Quadruple-tank process consists of four interconnected water tanks and two pumps as shown in Figure 1. The target is to control the level in the lower two tanks with two pumps [4]. The process inputs are $\mathrm{v}_{1}$ and $\mathrm{v}_{2}$ (input voltages to the pumps) and the outputs are $y_{1}=k_{c} h_{1}$ and $y_{2}=k_{c} h_{2}$ (voltages from level measurement devices). Mass balances and Bernoulli's law yield the following model:

$$
\begin{aligned}
& \frac{d h_{1}}{d t}=-\frac{a_{1}}{A_{1}} \sqrt{2 g h_{1}}+\frac{a_{3}}{A_{1}} \sqrt{2 g h_{3}}+\frac{\gamma_{1} k_{1}}{A_{1}} v_{1} \\
& \frac{d h_{2}}{d t}=-\frac{a_{2}}{A_{2}} \sqrt{2 g h_{2}}+\frac{a_{4}}{A_{2}} \sqrt{2 g h_{4}}+\frac{\gamma_{2} k_{2}}{A_{2}} v_{2} \\
& \frac{d h_{3}}{d t}=-\frac{a_{3}}{A_{3}} \sqrt{2 g h_{3}}+\frac{\left(1-\gamma_{2}\right) k_{2}}{A_{3}} v_{2} \\
& \frac{d h_{4}}{d t}=-\frac{a_{4}}{A_{4}} \sqrt{2 g h_{4}}+\frac{\left(1-\gamma_{1}\right) k_{1}}{A_{4}} v_{1}
\end{aligned}
$$

where, $\gamma_{i}$ is the flow distribution to lower and diagonal upper tank, $A_{i}$ is the cross-section area, $a_{i}$ is the outlet hole cross section and $h_{i}$ is the water level, in tank $i$ respectively.

The voltage applied to pump $i$ is $v_{i}$ and the corresponding flow is $k_{i} v_{i}$. The parameters $\gamma_{1}, \gamma_{2} \in(0,1)$ are determined from how the valves are set prior to an experiment. The flow to tank 1 is $y_{1} k_{1} v_{1}$ and the flow to tank 4 is $\left(1-y_{1}\right) k_{1} v_{1}$ and similarly for tank 2 and 3 . The acceleration of gravity is denoted by ' $\mathrm{g}$ '. This typical system has two finite zeros for $\gamma_{1}, \gamma_{2} \in(0,1)$ one always lies in the left half-plane, but, the other can be placed either in the left or the right half-plane depending on the valve setting of $\gamma_{1}, \gamma_{2}$ as explained in table $1,[5]$. 


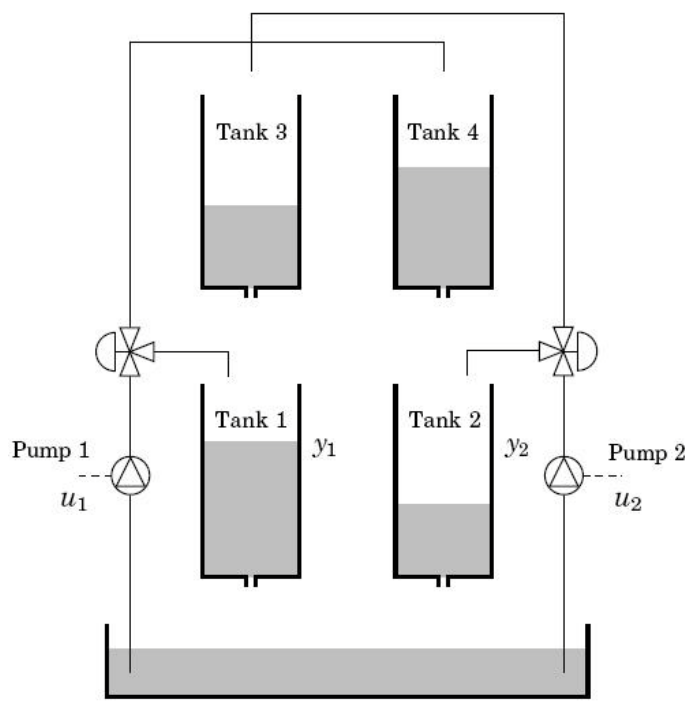

Fig. 1. Diagram for Quadruple Tank Process.

Table 1. For Valve Setting.

\begin{tabular}{|c|c|c|}
\hline Valve Settings & Process & Zero Location \\
\hline If $1<\gamma_{1}+\gamma_{2} \leq 2$ & $\begin{array}{c}\text { Minimum } \\
\text { Phase }\end{array}$ & $\begin{array}{c}\text { Zero is in left } \\
\text { plane }\end{array}$ \\
\hline If $0 \leq \gamma_{1}+\gamma_{2}<1$ & $\begin{array}{c}\text { Non } \\
\text { Minimum } \\
\text { Phase }\end{array}$ & $\begin{array}{c}\text { Zero is in left } \\
\text { plane }\end{array}$ \\
\hline If $\gamma_{1}+\gamma_{2}=1$ & & Zero at Origin \\
\hline
\end{tabular}

Linearised the model has two sets of operating points with state space equation at operating points $x_{i}=h_{i}-h_{i}^{0}$ and $u_{i}=v_{i}-v_{i}^{0}$.

$$
\begin{aligned}
\frac{d x}{d t}= & =\left[\begin{array}{cccc}
\frac{-1}{T} & 0 & \frac{A}{A T} & 0 \\
0 & \frac{-1}{T} & 0 & \frac{A}{A T} \\
0 & 0 & \frac{-1}{T} & 0 \\
0 & 0 & 0 & \frac{-1}{T}
\end{array}\right] x+\left[\begin{array}{cc}
\frac{\gamma k}{A} & 0 \\
0 & \frac{\gamma k}{A} \\
0 & \frac{(1-\gamma) k}{A} \\
\frac{(1-\gamma) k}{A} & 0
\end{array}\right] u \\
y & =\left[\begin{array}{cccc}
k_{c} & 0 & 0 & 0 \\
0 & k_{c} & 0 & 0
\end{array}\right] x
\end{aligned}
$$

Here the time constant is

$T_{i}=\frac{A_{i}}{a_{i}} \sqrt{\frac{2 h_{i}^{0}}{g}}$
The dynamics for the process transfer function matrix is

$$
G(s)=\left[\begin{array}{cc}
\frac{\gamma_{1} c_{1}}{\left(1+s T_{1}\right)} & \frac{\left(1-\gamma_{2}\right) c_{2}}{\left(1+s T_{3}\right)\left(1+s T_{1}\right)} \\
\frac{\left(1-\gamma_{1}\right) c_{2}}{\left(1+s T_{4}\right)\left(1+s T_{2}\right)} & \frac{\gamma_{2} c_{2}}{\left(1+s T_{2}\right)}
\end{array}\right]
$$

\section{DESCRIPTION OF DESIGN STRATEGY FOR STABILITY}

\subsection{Decentralized method}

The basic block diagram of quadruple tank is represented by Two Input Two Output (TITO) process as shown in fig.2. The transfer function matrix is [11]

$$
G(s)=\left(\begin{array}{ll}
G_{11}(s) & G_{12}(s) \\
G_{21}(s) & G_{22}(s)
\end{array}\right)
$$

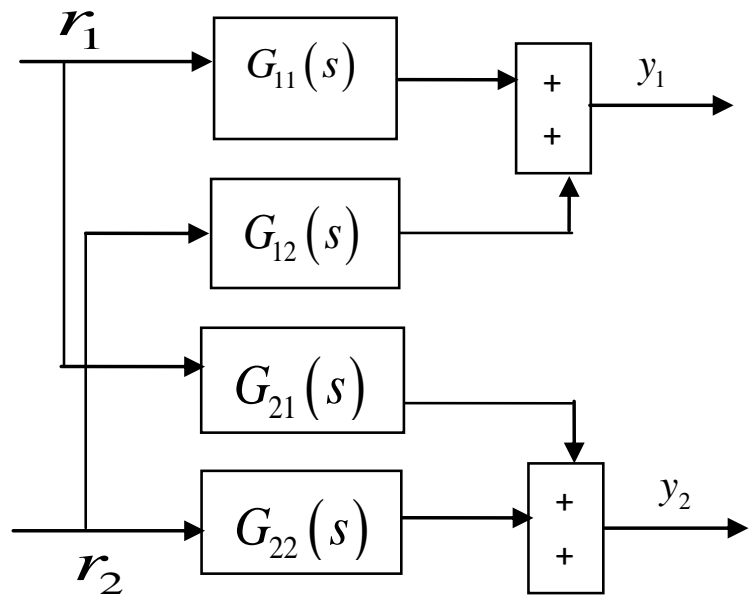

Fig. 2 Quadruple tank TITO process configuration

The diagonal elements $G_{12}$ and $G_{21}$ are interaction between two separate input-output pairs. The interaction between two loops produces different undesirable effects; such loops disturb each other or even destabilize the process. In quadruple tank process, interaction can prevent satisfactory control, with suitable pairing controllers. In this case, it is necessary to minimize the effect, by the use of decoupling structure so that desirable control can be achieved by separate single loop controller. The decouple method converts MIMO process into SISO system by eliminating interaction between loops [1]. Quadruple tank process in which channels are decoupled in the form of two cascade sub-processes, where the decoupler has the transfer matrix as shown in Figure 3, [2]. 


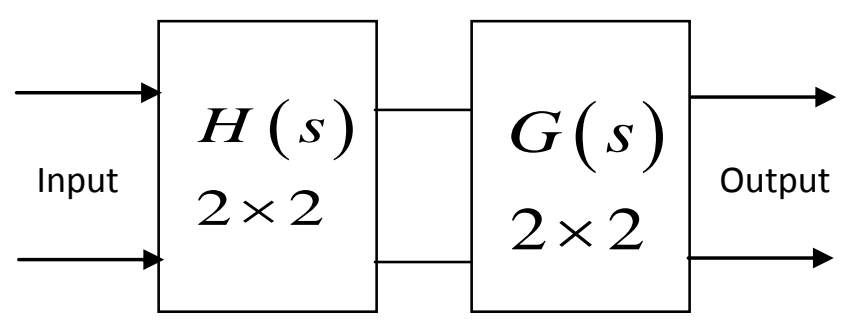

Fig: 3 Diagonalization of the quadruple tank using decoupler

The transfer matrix

$$
H(s)=\left(\begin{array}{ll}
H_{11}(s) & H_{12}(s) \\
H_{21}(s) & H_{22}(s)
\end{array}\right)
$$

The following equation should be

$\left[\begin{array}{ll}\mathrm{G}_{11}(\mathrm{~s}) & \mathrm{G}_{12}(\mathrm{~s}) \\ \mathrm{G}_{21}(\mathrm{~s}) & \mathrm{G}_{22}(\mathrm{~s})\end{array}\right]\left[\begin{array}{ll}\mathrm{H}_{11}(\mathrm{~s}) & \mathrm{H}_{12}(\mathrm{~s}) \\ \mathrm{H}_{21}(\mathrm{~s}) & \mathrm{H}_{22}(\mathrm{~s})\end{array}\right]=\left[\begin{array}{cc}\mathrm{G}_{\mathrm{n} 1}(\mathrm{~s}) & 0 \\ 0 & \mathrm{G}_{\mathrm{n} 2}(\mathrm{~s})\end{array}\right]$ (8)

This can be explained as,

$$
\begin{aligned}
& H_{11}(s) G_{11}(s)+H_{21}(s) G_{12}(s)=G_{n 1}(s) \\
& H_{12}(s) G_{21}(s)+H_{22}(s) G_{22}(s)=G_{n 2}(s) \\
& H_{11}(s) G_{21}(s)+H_{21}(s) G_{22}(s)=0 \\
& H_{12}(s) G_{11}(s)+H_{22}(s) G_{12}(s)=0
\end{aligned}
$$

From the equation (9) it is required to determine the decoupler transfer function. According to these equations s $H_{11}(s)=H_{22}(s)=1$ is chosen. Using eQn (10), (11) the other decoupler function is chosen as.

$$
\begin{aligned}
& \mathrm{H}_{12}(\mathrm{~s})=-\mathrm{G}_{12}(\mathrm{~s}) / \mathrm{G}_{11}(\mathrm{~s}) \\
& \mathrm{H}_{21}(\mathrm{~s})=-\mathrm{G}_{21}(\mathrm{~s}) / \mathrm{G}_{22}(\mathrm{~s})
\end{aligned}
$$

The quadruple tank process can be represented by the following input-output relation, as given in eqns. [12] and eqn. [13],

$\mathrm{y}_{1}=\left(\mathrm{G}_{11}(\mathrm{~s})-\frac{\mathrm{G}_{21}(\mathrm{~s})}{\mathrm{G}_{22}(\mathrm{~s})} \mathrm{G}_{21}(\mathrm{~s})\right) \mathrm{r}_{1}+0 * \mathrm{r}_{2}=\mathrm{G}_{\mathrm{n} 1}(\mathrm{~s}) \mathrm{r}_{1}$

$$
\mathrm{y}_{2}=0 * \mathrm{r}_{1}+\left(\mathrm{G}_{22}(\mathrm{~s})-\frac{\mathrm{G}_{12}(\mathrm{~s})}{\mathrm{G}_{11}(\mathrm{~s})} \mathrm{G}_{21}(\mathrm{~s})\right) \mathrm{r}_{2}=\mathrm{G}_{\mathrm{n} 2}(\mathrm{~s}) \mathrm{r}_{2}
$$

\subsection{Centralized method}

To evaluate the effect of control loop interactions for quadruple tank process as a conventional multiloop (TITO) control scheme consisting of two feedback controllers is to be studied.
Figure 4 indicates that the process interactions that can induce undesirable interactions between the control loops. Two possible control configurations are shown in fig 4 . One is direct effect on $y_{1}$ as controlled by $u_{1}$, while $y_{2}$ is controlled by $u_{2}$.the alternative strategy is to pair $y_{1}$ with $u_{2}$ and $y_{2}$ with $u_{1}$.

Deriving the following expression related to controlled variable and set points [11]:

$$
\begin{aligned}
& y_{1}=\Gamma_{11} u_{1}+\Gamma_{21} u_{2} \\
& y_{2}=\Gamma_{11} u_{1}+\Gamma_{21} u_{2}
\end{aligned}
$$

Where the closed loop transfer functions are

$$
\begin{aligned}
& \Gamma_{11}=\frac{G_{11}+\left(G_{11} G_{22}-G_{12} G_{21}\right)}{\Delta(S)} \\
& \Gamma_{12}=\frac{G_{12}}{\Delta(S)} \\
& \Gamma_{21}=\frac{G_{21}}{\Delta(S)} \\
& \Gamma_{21}=\frac{G_{22}+\left(G_{11} G_{22}-G_{12} G_{21}\right)}{\Delta(S)}
\end{aligned}
$$

And $\Delta(S)$ is defined as

$$
\Delta(S)=\left(1+G_{11}\right)\left(1+G_{22}\right)-G_{12} G_{21}
$$

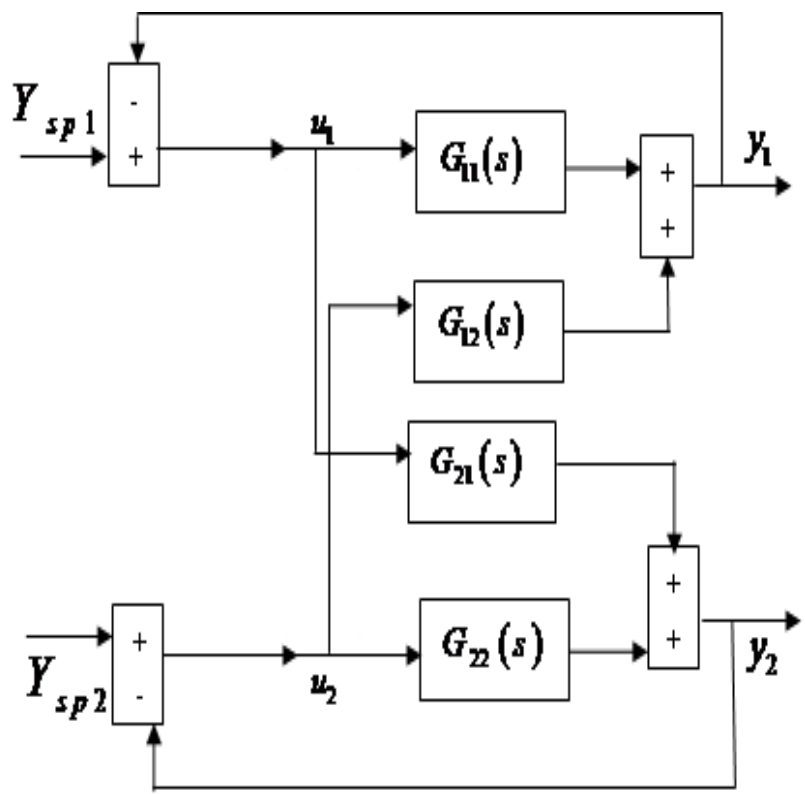

Fig. 4: Block diagram of interactive process. 


\section{DESCRIPTION OF MPC}

In MPC applications, the output variables are also referred to as controlled variables or CV's, while the input variables are called as manipulated variables or MV's. The predictions are made in two types of MPC calculations that are performed at each sampling instant: set-point calculations [6] and control calculations. Inequality constraints as upper and lower limits can be included in either types of calculation [7].

\subsection{Basic description}

In MPC the set points are typically calculated each time for MIMO process with $u$ input variables and $y$ output variables

The current values of $u$ and $y$ as $u(k)$ and $y(k)$. The objective is to calculate the optimum set point $y_{s p}$ for the next control calculation (at $k+1)$ and also to determine the corresponding steady-state value of $u, u_{s p}$. This value is used as the set point for $u$ for the next control calculation [12].

A general, linear steady-state process model can be written as [11]

$$
\Delta y=K \Delta u
$$

Where $K$ is the steady-state is gain matrix and $\Delta u, \Delta y$ denotes steady-state changes in $u$ and $y$ it is convenient to define $\Delta u$ and $\Delta y$ as

$$
\Delta y=y_{s p}-y O L(k)
$$

Here $y O L(k)$ is the steady-state value of $y$

$$
\Delta u=u_{s p}-u(k)
$$

To include incorporate output feedback, the steady-state model Eqn (21) is modified as

$$
\Delta y=K \Delta u+[y(k)-\overline{y(k)}]
$$

In general, the model predictive control problem is formulated to solve a finite horizon open-loop optimal control problem subject to system dynamics and constraints involving states and controls. Fig. 5 shows the basic principle of model predictive control. The measurements are obtained from plant at regular intervals at time $k$, The controller predicts the future dynamic behavior of the system over a predicted horizon $\mathrm{P}$ and control horizon $\mathrm{M}$ determines (over a control horizon $\mathrm{P}>\mathrm{M}$ ) the input such that a predetermined open-loop as well as closed-loop performance objective functional is optimized. If there were no disturbances and no model-plant mismatch, and if the optimization problem could be solved for infinite horizons, then one could apply the input function found at time $k=0$ to the system for all times $k \geq 0$. However, this is not possible in general. Due to disturbances and modelplant mismatch, the true system behavior is different from the predicted behavior [6]. Here we have ignored disturbances and match plant model.

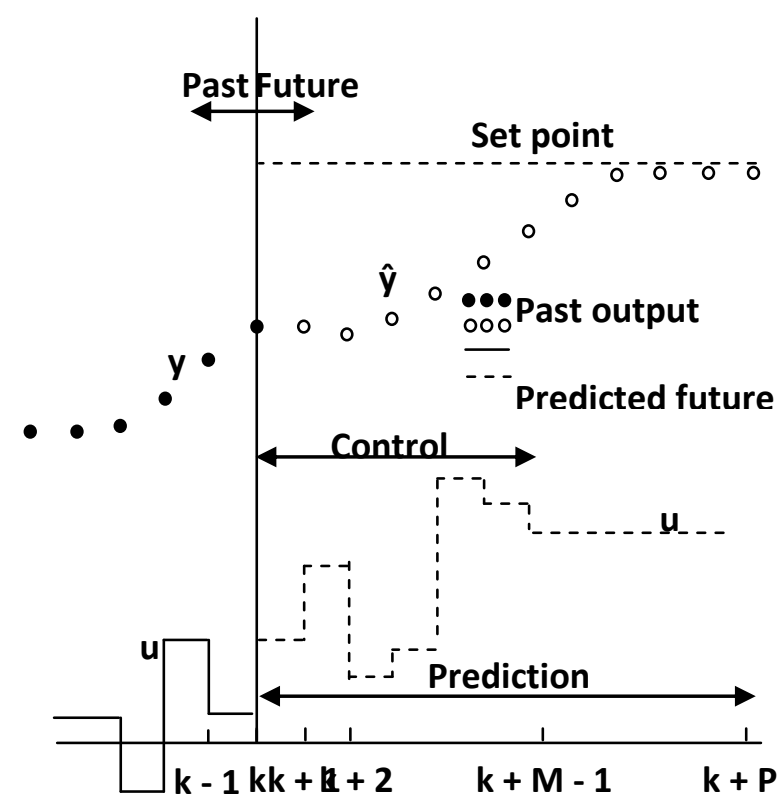

Fig. 5: Basic principle of Model Predictive Control.

\subsection{Decentralized model predictive control}

The MPC algorithm consists of main term for optimization of unequal constraints [12]:

The least square objective and Model

4.2.1 The least square objective

The least square objective function is written for decentralized or SISO (Single Input and Single Output) [12]

$\Phi=\sum_{i=1}^{P}\left|r_{k+i}-\hat{y}_{k+i}\right|+w \sum_{i=0}^{M-1}\left|\Delta u_{k+i}\right|$

$P$ - Predictive control

$M-$ Control horizon

$\hat{y}$ - Predictive output

$r-$ Set point

$\Delta u-$ Change in manipulated input

$k$-Sample time

\subsubsection{Model}

In MPC two types of models are used for predicted output, which are discrete models. These are Finite Step Response (FSR) and Finite Impulse Response (FIR)

FSR models are obtained by making a unit step input change to a process operation at steady state. The step response model is the vector of step response coefficients.

$S=\left[s_{1} s_{2} s_{3} \ldots . . s_{N}\right]$

$\mathrm{N}=$ model length 


\subsection{Centralized model predictive control}

An MPC algorithm consists of:

Cost Function, Constraint and a Model of the Process.

\subsubsection{Cost Function}

The main idea in MPC is that the MPC controller calculates a sequence of future control actions such that the cost function is minimized.

The cost function often used in MPC is like this (a linear quadratic function)[11]:

$J=\sum_{k=0}^{N_{p}}(\hat{y}-r)^{T} Q(\hat{y}-r)+\sum_{k=0}^{N_{p}} \Delta u^{T} R \Delta u$

Where:

$N_{p}$ - Prediction horizon

$r-$ Set point

$\hat{y}-$ Predicted process output

$\Delta u$ - Predicted change in control value, $\Delta u_{k}=u_{k}-u_{k-1}$

$Q$ - Output error weight matrix

$R-$ Control weight matrix

This works for MIMO systems (Multiple Input and Multiple Outputs) so we are dealing with vectors and matrices.

\subsubsection{Constraints}

All physical systems have constraints. Generally, physical constraints like actuator and valve limits, etc and performance constraints like overshoot, settling time, etc. In MPC one normally defines these constraints [11] to minimize inequalities.

Constraints in the outputs:

$y_{\min } \leq y \leq y_{\max }$

Constraints in the inputs:

$\Delta u_{\min } \leq \Delta u \leq \Delta u_{\max }$

$u_{\min } \leq u \leq u_{\max }$

Note: $\Delta u_{k}=u_{k}-u_{k-1}$

\subsubsection{Model}

The main drawback with MPC is that a model for the process, i.e., a model which describes the input to output behavior of the process, is needed. Mechanistic models derived from conservation laws can be used. Usually, however in practice simply data-driven linear models are used. We consider the stabilization problem for a class of systems described by the following nonlinear of differential equations [9]:

$$
\begin{aligned}
& \dot{x}(t)=A(t) f[x(t), u(t)], t \geq 0, x\left(t_{0}\right)=x_{0}, \\
& x \in X \subset R^{c}, u \in U \subset R^{m}, A(.): R_{\geq 0} \rightarrow R^{c \times m}
\end{aligned}
$$

With the known smooth nonlinear map $f[x(t), u(t)]$ and the unknown parameter matrix $A(t)$

\section{SIMULATION ANALYSIS}

In this paper, simulation results are compared with [1], [2] and [3] on time based domain using tuning predictive control response, to a step response input. Here, response is plotted for the lower tanks for minimum and non minimum phase at two operating points at $p_{-}$and $p_{+}$of minimum and non minimum phase [10]. These operating points are at Table.2.

Table 2. Operating Points.

\begin{tabular}{|c|c|c|c|}
\hline $\begin{array}{c}\text { Operating } \\
\text { Points }\end{array}$ & Units & $p_{-}$ & $p_{+}$ \\
\hline$\left(h_{1}^{0}, h_{2}^{0}\right)$ & {$[\mathrm{cm}]$} & $(12.4,12.7)$ & $(12.6,13.0)$ \\
\hline$\left(h_{3}^{0}, h_{4}^{0}\right)$ & {$[\mathrm{cm}]$} & $(1.8,1.4)$ & $(4.8,4.9)$ \\
\hline$\left(v_{1}^{0}, v_{2}^{0}\right)$ & {$[\mathrm{V}]$} & $(1,1)$ & $(1,1)$ \\
\hline$\left(k_{1}, k_{2}\right)$ & {$\left[\mathrm{cm}^{3} / \mathrm{Vs}\right]$} & $(3.33,3.35)$ & $(3.14,3.29)$ \\
\hline$\left(\gamma_{1}, \gamma_{2}\right)$ & & $(0.7,0.6)$ & $(0.43,0.34)$ \\
\hline
\end{tabular}

\subsection{Decentralized response}

Decentralized response is plotted for quadruple tank process of lower two tanks. For minimum phase response of lower tank output $\mathrm{y} 1$ with specified input $\mathrm{u} 1$ and output $\mathrm{y} 2$ with specified input $\mathrm{u} 2$ as shown in fig 6,7 with $\mathrm{P}=8, \mathrm{M}=1$ and model length 50

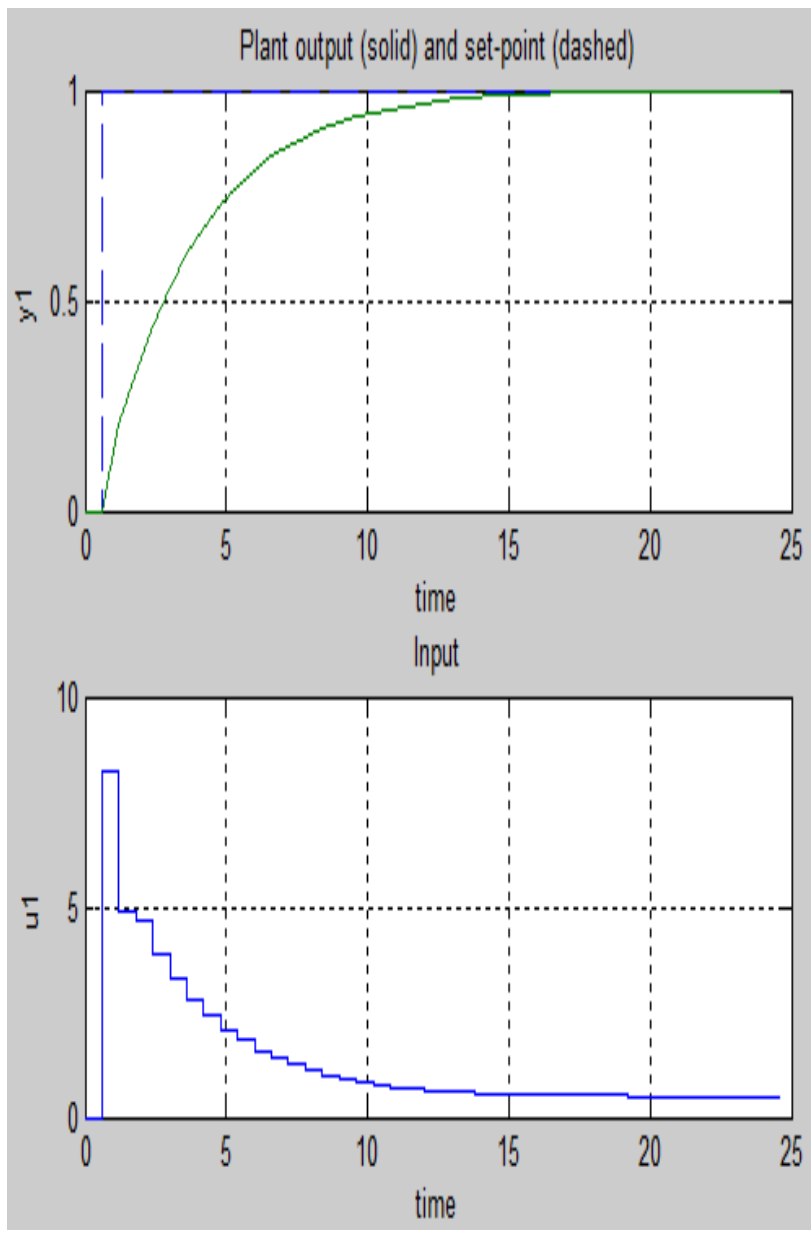

Fig.6 Output y1response with specified input u1 


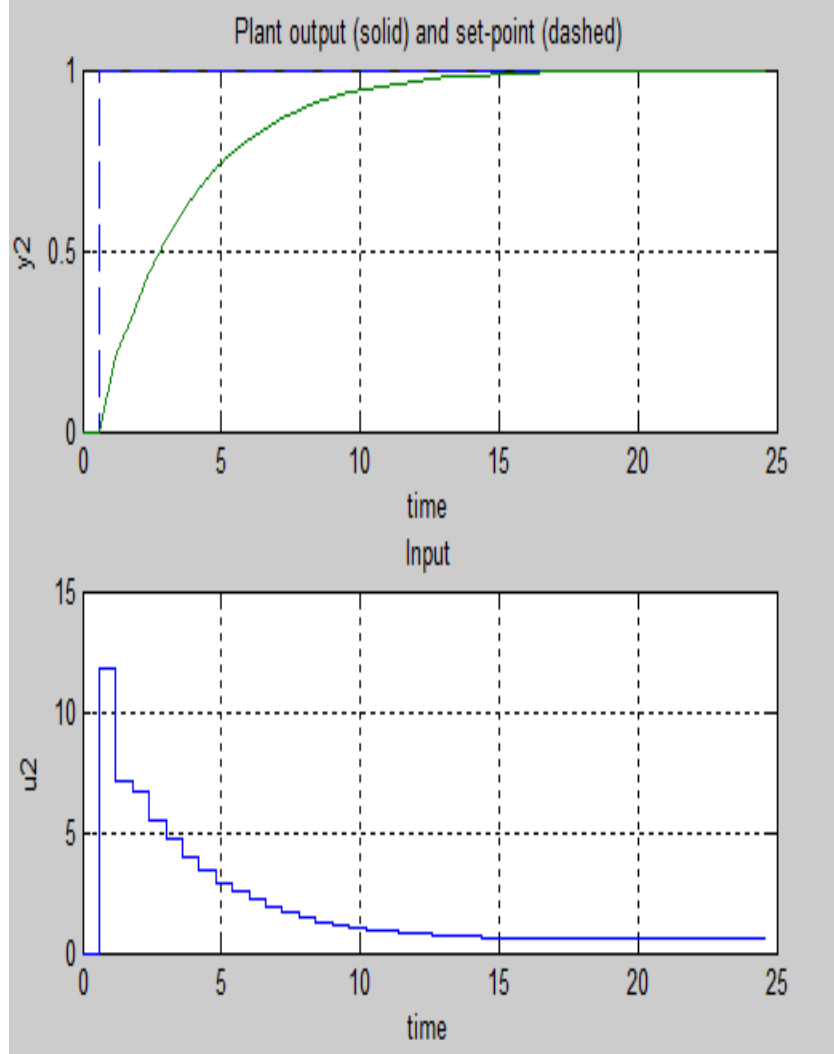

Fig.7 Output y2 response with specified input $\mathrm{u} 2$

For non minimum phase response of lower tank output y1 with specified input $\mathrm{u} 1$ and output $\mathrm{y} 2$ with specified input $\mathrm{u} 2$ as shown in fig 8,9 with $\mathrm{P}=8, \mathrm{M}=1$ and model length 50

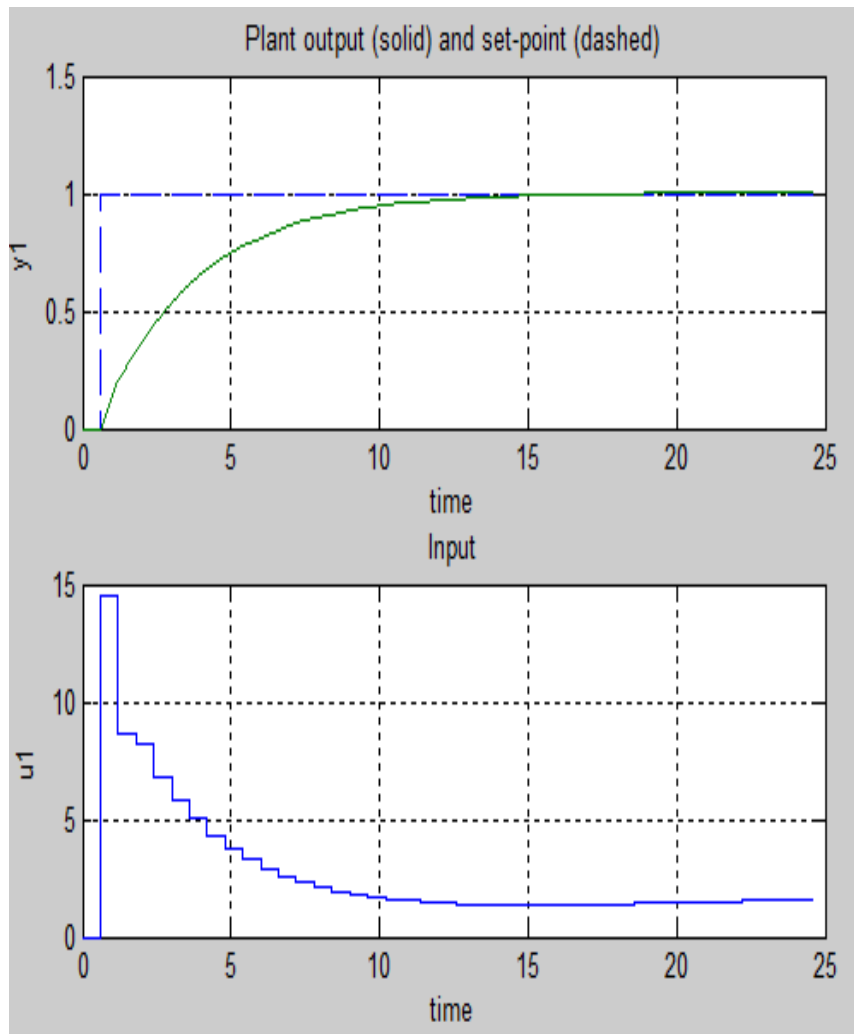

Fig.8 Output y1 response with specified input u1

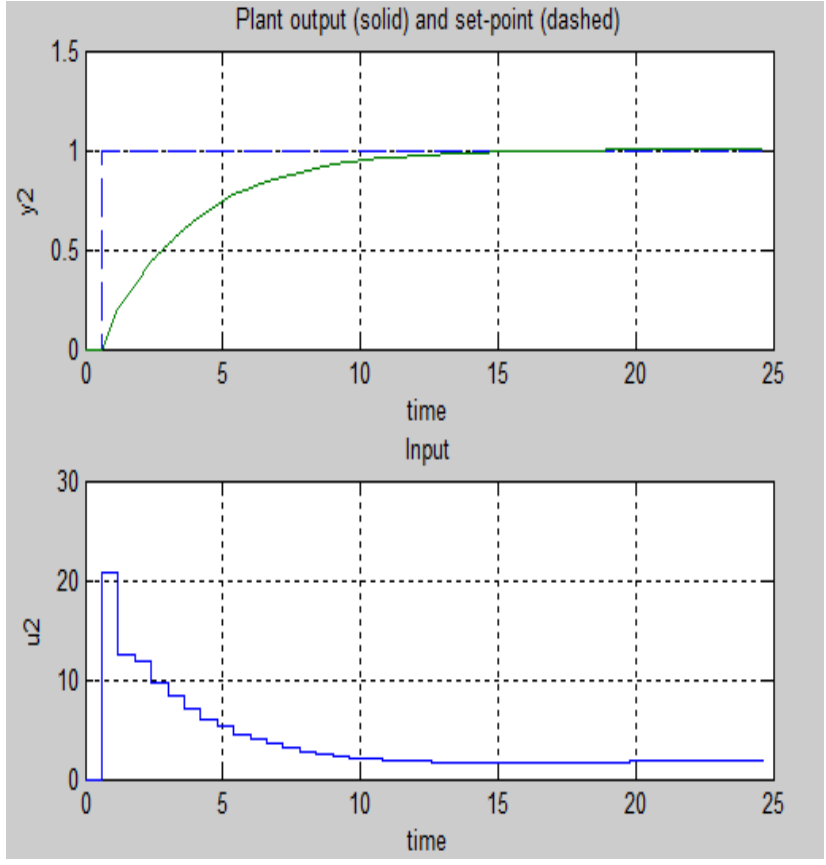

Fig.9 Output y2 response with specified input u2

\subsection{Centralized response}

Response has plotted centralized of quadruple tank process of lower two tanks. For minimum phase response of lower tank output $\mathrm{y} 1$ with specified input $\mathrm{u} 1$ as shown in fig 10 with $\mathrm{P}=10, \mathrm{M}=3$ and control interval $=0.25$ and lower tank output $\mathrm{y} 1$ with specified input $\mathrm{u} 2$ as shown in fig 11 with $\mathrm{P}=12, \mathrm{M}=3$ and control interval $=0.25$

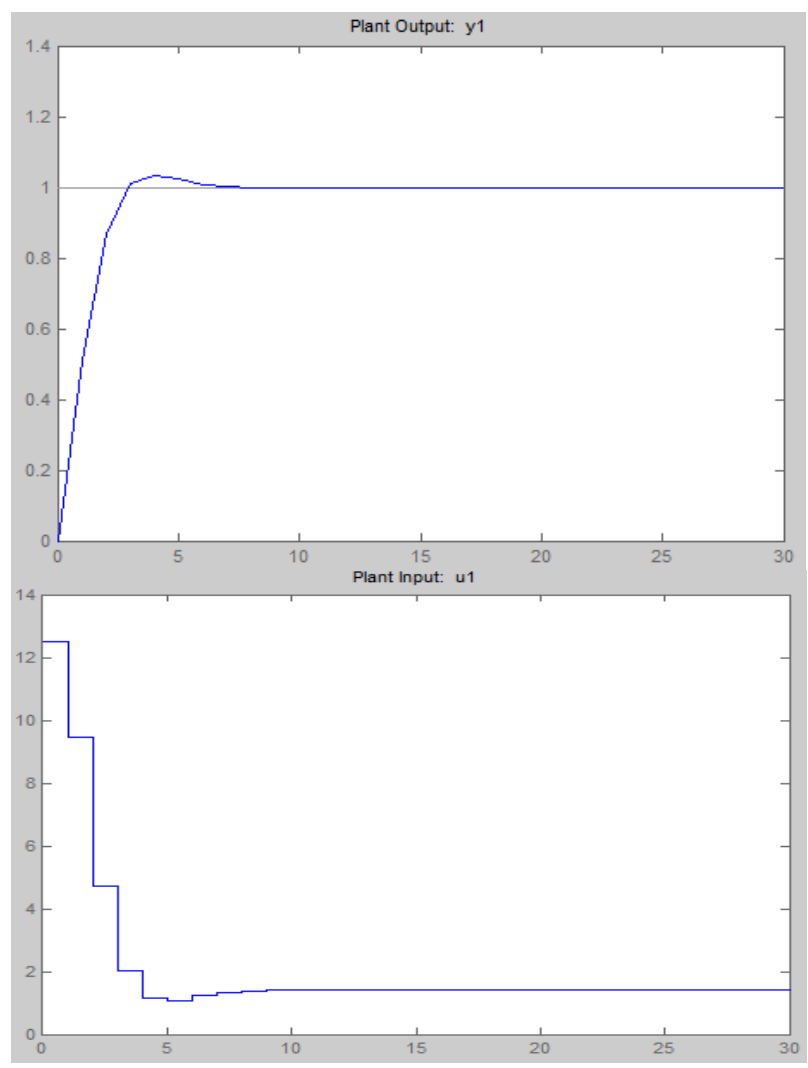

Fig.10 Output y1response with specified input u1 


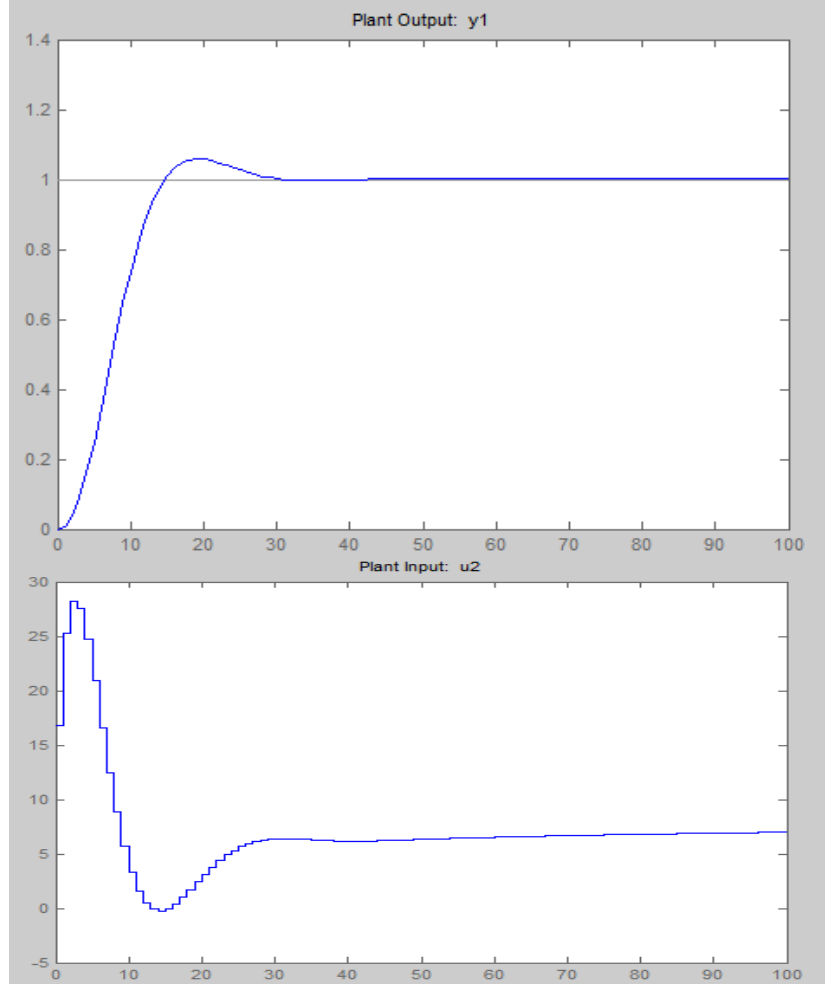

Fig.11 Output y1 response with specified input u2

For minimum phase response of lower tank output y2 with specified input $\mathrm{u} 1$ as shown in fig 12 with $\mathrm{P}=15, \mathrm{M}=3$ and control interval $=0.25$ and lower tank output $\mathrm{y} 2$ with specified input $\mathrm{u} 2$ as shown in fig 13 with $\mathrm{P}=10, \mathrm{M}=2$ and control interval $=0.25$

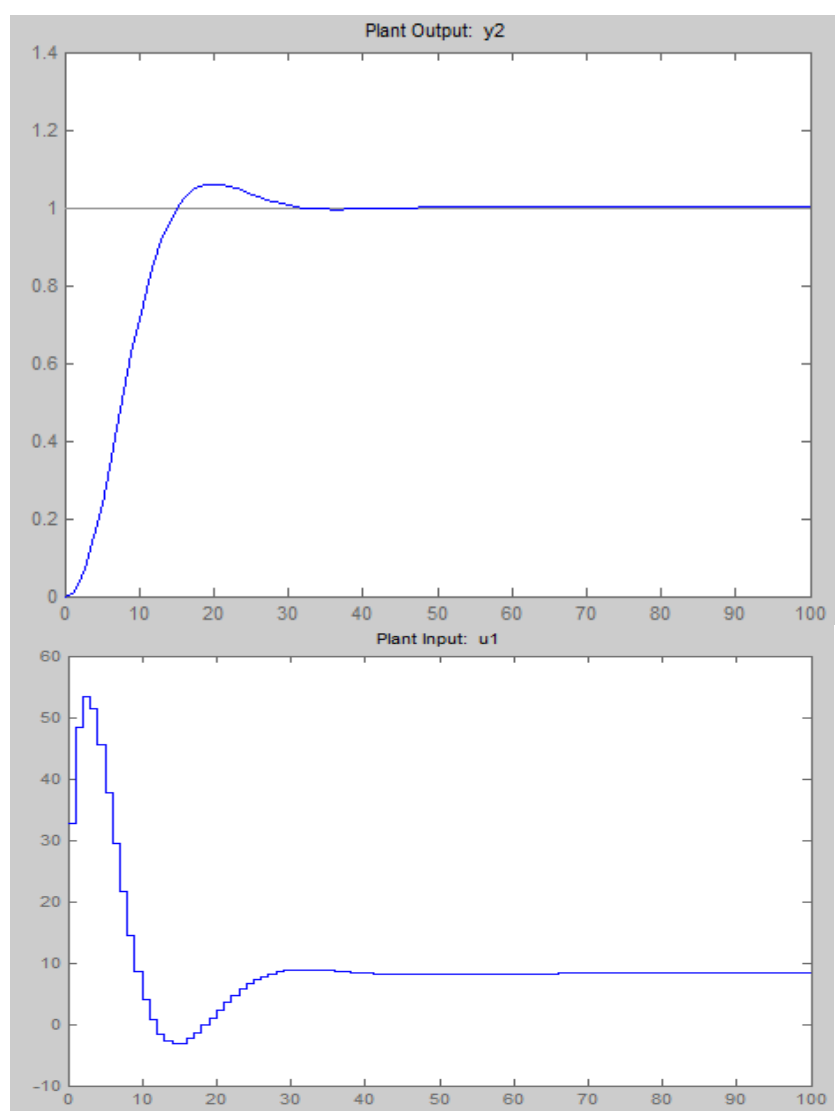

Fig.12 Output y2 response with specified input u1

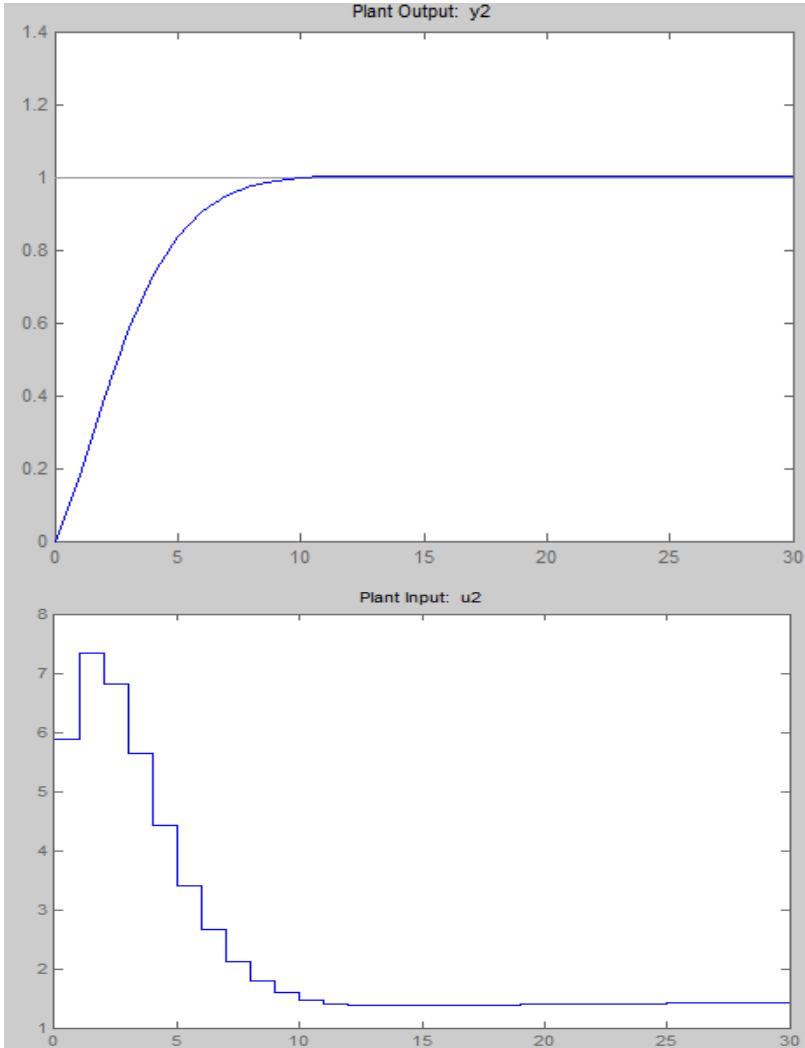

Fig.13 output $y 2$ response with specified input $u 2$

For non minimum phase response of lower tank output y1 with specified input $\mathrm{u} 1$ as shown in fig 14 with $\mathrm{P}=10, \mathrm{M}=2$ and control interval $=0.25$ and lower tank output $\mathrm{y} 1$ with specified input $\mathrm{u} 2$ as shown in fig 15 with $\mathrm{P}=15, \mathrm{M}=3$ and control interval $=0.25$

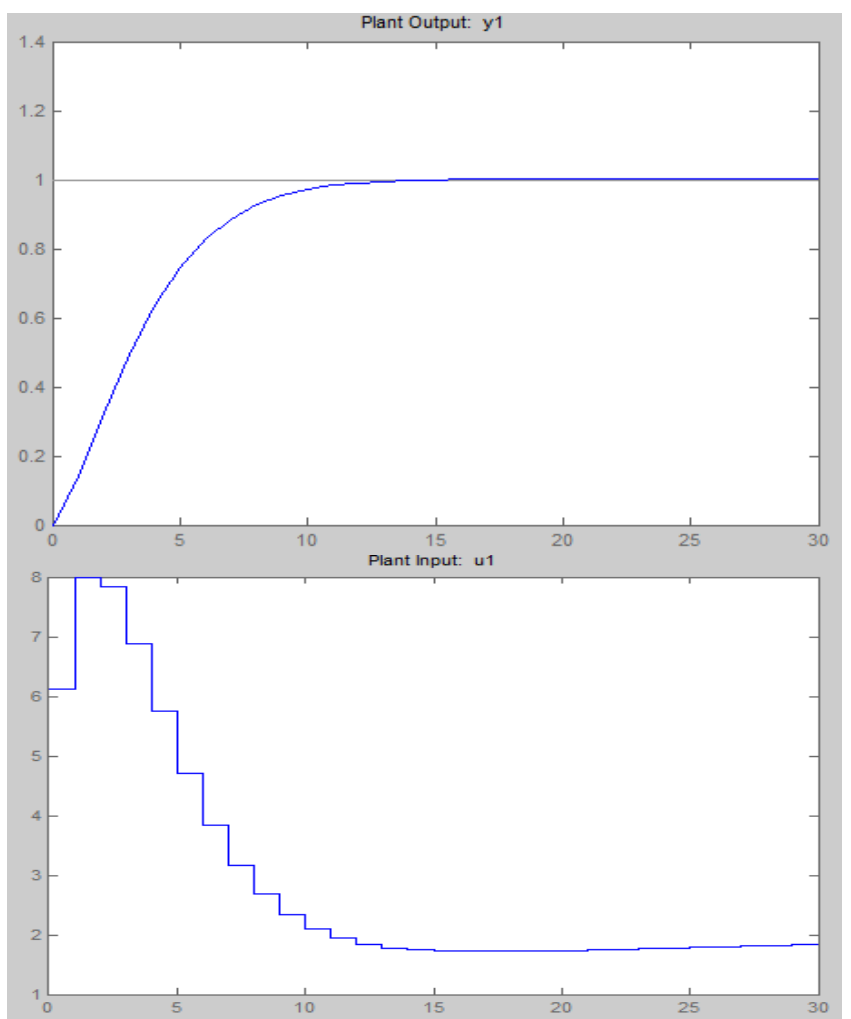

Fig. 14 output y1 response with specified input u1 


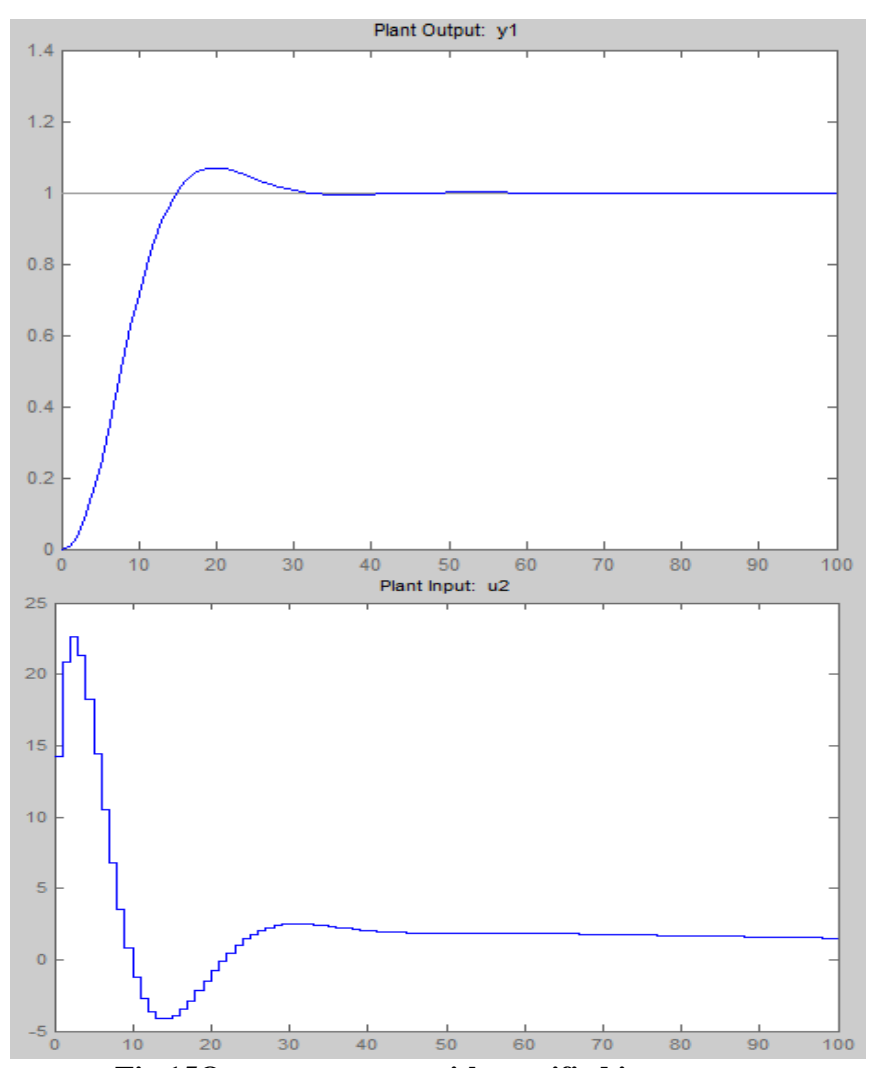

Fig.15Output response with specified input

For non minimum phase response of lower tank output y2 with specified input $u 1$ as shown in fig 16 with $\mathrm{P}=15, \mathrm{M}=3$ and control interval $=0.25$ and lower tank output $\mathrm{y} 2$ with specified input $\mathrm{u} 2$ as shown in fig 17 with $\mathrm{P}=10, \mathrm{M}=2$ and control interval $=0.25$

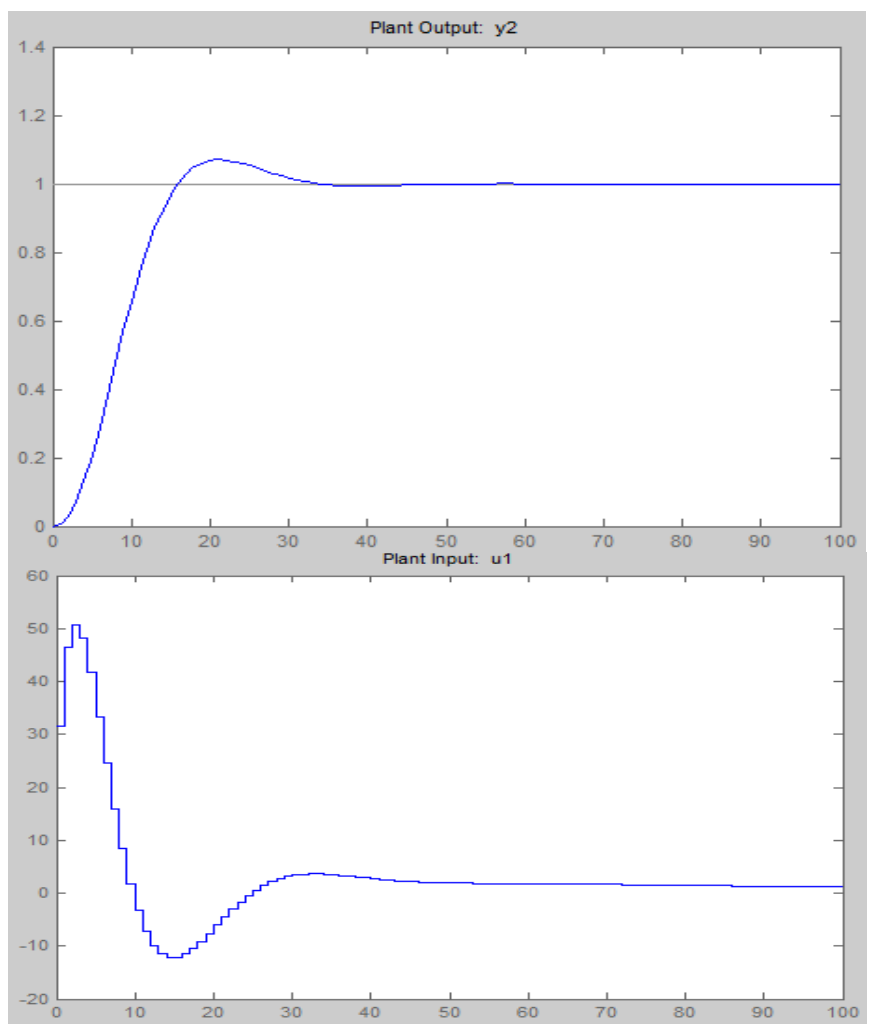

Fig. 16 Output y2 response with specified input u1

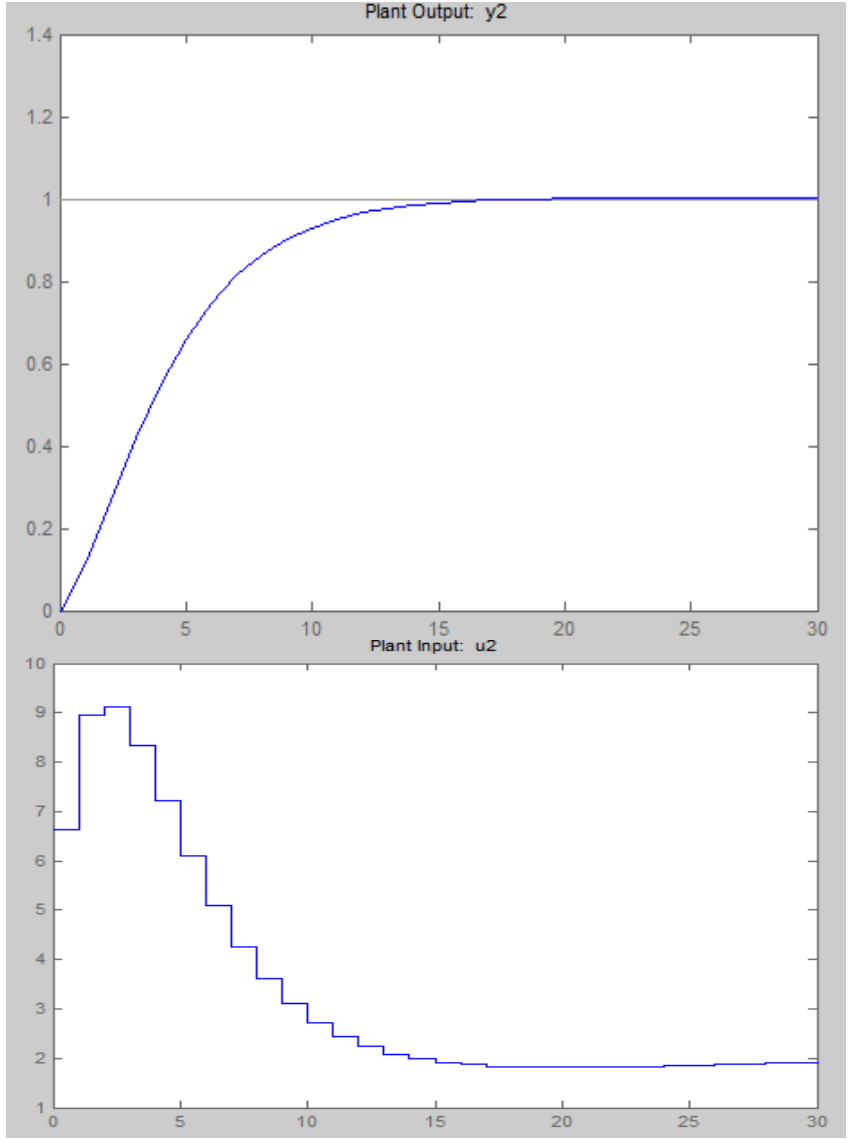

Fig.17 Output y2 response with specified input u1

\section{CONCLUSION}

The design procedure for MPC controller for Quadruple tank process has been proposed in this paper. The step response for process is compared with the results obtained in references listed at paper1, 2 and paper 3 for both minimum and non minimum phase of decentralized and centralized technique. It is observed that decentralized and centralized process with different controller parameters offers stable response for step input. Decentralized system exhibits stable response without overshoots for minimum and where as for non minimum phase without compensation exhibits stable response without overshoots. Similarly centralize systems also exhibit stable response without overshoot for output $\mathrm{y} 1$ with input $\mathrm{u} 1$ and output $\mathrm{y} 2$ with input $\mathrm{u} 2$ only for minimum and non minimum phase. Interchanging of inputs it exhibits overshoot stable response for both phases. Non minimum phase system has a transmission zero on right plane still exhibits stable response without any compensation for linear and non linear system. MPC is a more advanced technique to handle multivariable parameters. Finally, all transient and steady state responses have been obtained in all cases.

\section{REFERENCES}

[1] P. Srinivasarao and Dr. P. Subbaiah, "Decoupled control strategy for quadruple tank process," CIIT International Journal of Programmable Circuit and System, Vol. 4, No. 6, pp. 287-296, May 2012.

[2] P. Srinivasarao and Dr. P. Subbaiah, "Comparative study for Quadruple Tank Process with Coefficient Diagram Method," IFRSA International Journal of Electronics Circuits and System, Vol. 1, Issue 2, pp. 9099, July 2012. 
[3] P. Srinivasarao and Dr. P. Subbaiah, "Linear and Non linear Model Predictive Control of Quadruple Tank Process," International Journal of Electronics Circuits and System, Vol. 66, Issue 20, pp. 28-34, March 2013.

[4] K. H. Johansson, "The Quadruple-Tank Process: A Multivariable Laboratory Process with an Adjustable Zero," IEEE Transactions on Control Systems Technology, pp. 456-465, 2000.

[5] Qamar Saeed, Vali Uddin and Reza Katebi, "Multivariable Predictive PID Control for Quadruple Tank," World Academy of Science, Engineering and Technology,2010.

[6] M. Alamir, G. Bornard, "Stability of a truncated infinite constrained receding horizon scheme: the general discrete nonlinear case," Elsevier Science Ltd , Vol. 31, No. 9, pp. 1353-1356, Sep 1995.

[7] Zoltan K.Nagy Richard D.Braatz, "Roboust Nonlinear Model Predictive Control of Batch Processses", AIChe Journal, Vol. 49 No.7, July 2003.

[8] M. J. Lengare, R. H. Chile, L. M. Waghmare and Bhavesh Parmar, "Auto Tuning of PID Controller for MIMO Processes," World Academy of Science,
Engineering and Technology, 2008.

[9] Sergey Edward Lyshevski, Control system theory with Engineering Applications, Jaico Publishers, 2003.

[10] Ashish Tewari, Modern Conrol Design with MATLAB and SIMULINK, John Willy \& Sons, Ltd, 2009.

[11] Dale E. Seborg,Thomas F. Edgar and Duncan A.Mellichamp , Process Dynamics and Control, John Willy \& Sons, Ltd, 2006.

[12] B.Wayane Bequette, Process Control Modeling, Design and Simulation, Prentice Hall,2003.

\section{AUTHOR'S PROFILE}

P. Srinivasarao working as Associate Professor in VITS Group of Institutions, Sontyam, Visakhapatnam. Completed M.Tech from COE Pune, Ph.D Scholar, Dr. M.G..R. University.

Dr.P.Subbaiah is Professor in Dhanlaxmi college of Engineering, Thambaram, Chennai, Tamilnadu, India. 